\title{
EL DIALOGO INTERCULTURAL EN SALUD, NECESARIO PARA EL ABORDAJE DE LAS INEQUIDADES SANITARIAS Y LOS CONFLICTOS SOCIALES EN LA SELVA PERUANA. 2011
}

\author{
Gualberto Segovia ${ }^{1}$, Roberto Quispe ${ }^{2}$, Igor A. Segovia ${ }^{3}$
}

\footnotetext{
${ }^{1}$ Médico de Familia. Centro Nacional de Salud Intercultural. INS. Lima, Perú.

${ }^{2}$ Bach. Economía. Centro Nacional de Salud Intercultural. INS. Lima, Perú.

${ }^{3}$ Estudiante de Medicina. Universidad Ricardo Palma. Lima, Perú
}

\section{RESUMEN}

Se realizó el abordaje al problema sanitario, dentro de un problema social "derrame de petróleo" en la selva peruana. Con una serie de intervenciones del Diálogo Intercultural en Salud (DICS), estrategia educativa y de gestión de la Atención Primaria de la Salud (APS). Intervenimos en comunidades nativas de Canaán y Nuevo Sucre, participaron representantes del sector salud, representantes de la medicina tradicional, autoridades de las comunidades nativas (Apus) y representantes de instituciones públicas del distrito de Contamana - Loreto. Se identifica problemas en el acceso a los servicios de salud por problemas de geográficos, culturales, de financiamiento de las prestaciones y en especial de organización del servicio de salud local. Débil atención integral de salud que afecta los diagnósticos oportunos y un débil trabajo en prevención de enfermedades y promoción de la salud. Se valida el diseño general y los pasos del DICS en comunidades nativas.

Palabras claves: Promoción de la salud, Atención primaria de la salud, Prevención de enfermedades. (fuente: DeCS BIREME)

\section{THE INTERCULTURAL DIALOG IN HEALTH, WHICH IS NECESSARY FOR THE APPROACH OF HEALTH INEQUITIES AND SOCIAL CONFLICTS IN THE PERUVIAN JUNGLE. 2011}

\begin{abstract}
It was realized an approach to the health problem, within a social problem "oil spill" in the Peruvian jungle. With a series of interventions of Intercultural Dialog in Health (DISC' file), educational strategy and management of Primary Health Care $(\mathrm{PHC})$. We intervene in native communities of Canaan and New Sucre, attended by representatives of the health sector, representatives of traditional medicine, authorities of the native communities (Apus) and representatives of public institutions of the district of Contamana - Loreto. It is identifies problems in access to health services by problems of geographic, cultural, financing of benefits and in particular of organization of the local health department. Weak comprehensive health care that affect the timely diagnostics and a weak work in disease prevention and health promotion. It validates the general design and the steps of the DISC' file in native communities.
\end{abstract}

Key Words: Health Promotion, Primary Health Care , Disease Prevention. (source: MeSH NLM)

\section{INTRODUCCIÓN}

La inequidad sanitaria es un tema que afecta a todos nuestros países y en la que influyen en forma considerable el sistema económico y político mundial.

La Comisión sobre Determinantes Sociales de la Salud señala que la mala salud de los pobres, el gradiente social de salud dentro de los países y las grandes desigualdades sanitarias están provocados por una distribución desigual del poder, de los ingresos, los bienes y los servicios. Esa distribución desigual es resultado de una nefasta combinación de políticas y programas sociales deficientes, arreglos económicos injustos y una mala gestión política ${ }^{(1)}$

Por otro lado, un conflicto social debe ser entendido como un proceso complejo, en el cual sectores de la sociedad, el Estado y las empresas perciben que sus objetivos, intereses, valores o necesidades son contradictorios, y esa contradicción puede derivar en violencia. Por lo tanto, un conflicto es inherente a la vida, es una característica natural e inevitable de la existencia humana y de la interacción social ${ }^{(2)}$.

Realizamos un análisis de sistematización de la estrategia del diálogo intercultural en salud, como un medio para mejorar la gestión de la atención primaria de la salud, aprovechando un conflicto social en la selva peruana y promover una gestión más participativa de los servicios de salud, tal como lo detalla el convenio 169 de la OIT, "Estos servicios deberán planearse y administrarse en coordinación con los pueblos interesados" (3). 


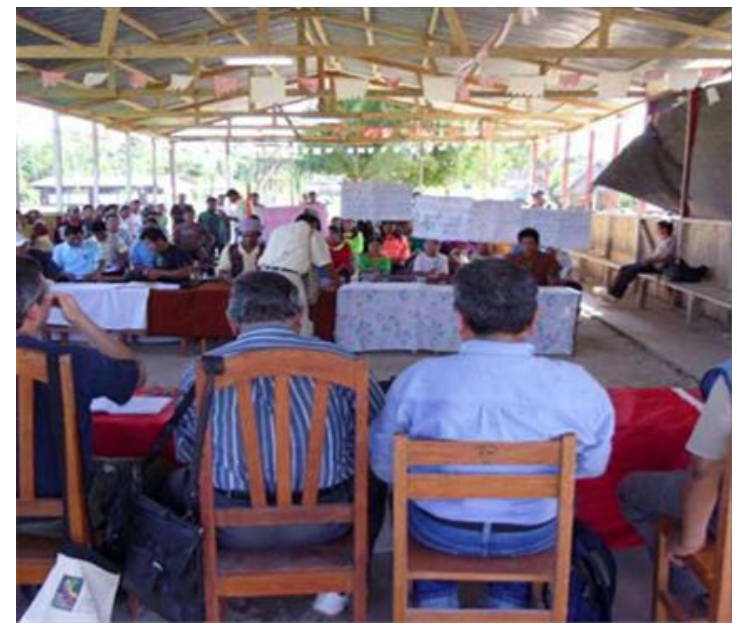

Figura 1. Reunión de la Comunidad Nativa Nuevo Sucre y reunión con representantes de la Comisión Nacional.

\section{MATERIAL Y MÉTODOS}

El presente estudio es el reporte de una intervención de tipo cualitativa, utilizando el Diálogo Intercultural en Salud (DICS) como una herramienta educativa y de gestión para la construcción de relaciones de articulación y acuerdos, entre representantes del sistemas de salud académico, de la medicina tradicional, representantes de las comunidades nativas e instituciones locales; con la finalidad de validar el DICS y desarrollar los procesos creativos que permita el abordaje de los determinantes sociales y culturales de la salud. ${ }^{(4)} \mathrm{La}$ intervención se realizó como consecuencia del derrame de petróleo en la comunidad nativa de Canaán de Cachiyacu y Nuevo Sucre en la Provincia de Ucayali (Loreto).

\section{RESULTADOS}

El Centro Nacional de Salud Intercultural participa en la Comisión Multisectorial sobre el derrame de petróleo en las comunidades nativas (CCNN) de Canaán de Cachiyacu y Nuevo Sucre, ambas comunidades ShipiboConibo de la provincia de Ucayali, del distrito de Contamana - Loreto desde agosto del 2011 hasta Octubre del $2011^{(5)}$.

Canaán de Cachiyacu ("Canaán") y Nuevo Sucre son comunidades nativas con 1,907 habitantes. Antes de 1975, la comunidad de Canaán estaba localizada al otro lado del río Ucayali, pero fue necesario trasladarla debido a la desviación del curso del río. Desde 1975, la comunidad ocupa su ubicación actual. La concesión de petróleo en esta área es el Lote 31-B y las actividades de la empresa Maple Gas se iniciaron en 1994, cuando Maple se hizo cargo de las operaciones de Petro Perú. Desde el 2001 Maple comienza sus operaciones en tierras de la Comunidad de Nuevo Sucre en el lote $31-E^{(6)}$.

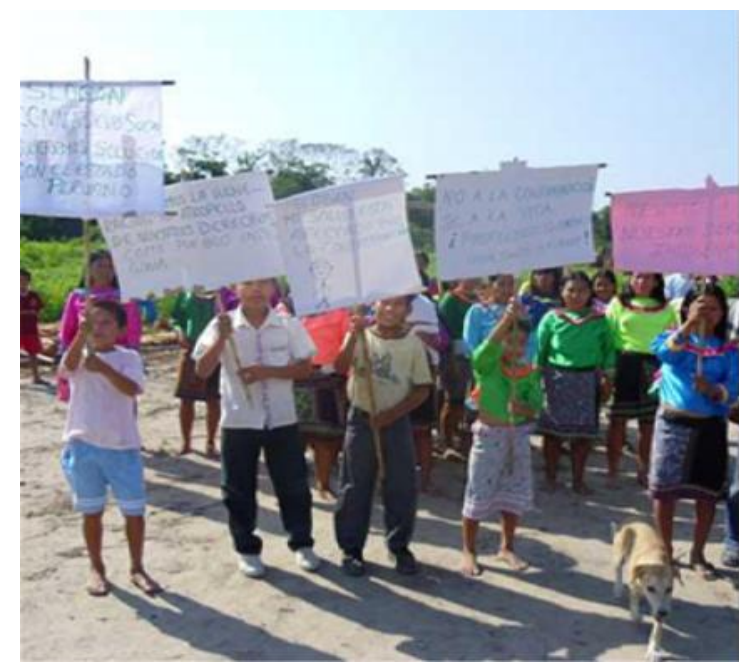

Figura 2. Protesta en caso derrame de petróleo. Nuevo Sucre - Contamana. 2011.

Los resultados del diálogo intercultural en salud con los representantes de las comunidades nativas Shipibo Conibo de Canaán y Nuevo Sucre, representantes del gobierno central (representantes del Ministerio de Salud, Ministerio del Ambiente), gobierno local (Municipalidad de Contamana) y del servicios de salud (Centro de Salud Contamana a la vez cabecera de la microrred); se pudo rescatar información válida para la toma de decisiones en la mejora de la situación de salud de las comunidades nativas. Han transcurrido 17 años de explotación petrolera y se percibe una creciente demanda por los servicios de salud; ambas comunidades nativas no disponen de agua potable ni desagüe; no hay un plan de letrinización; el fluido eléctrico es limitado a horas; se evidencia falta de documento de identidad en la población pobre y en consecuencia falta de acceso al seguro integral de salud; con una pobreza que llega al $60 \%$ de los habitantes en el distrito y que debe ser mayor en las comunidades nativas visitadas.

El financiamiento de la salud se realiza a través del SIS, pero en las comunidades nativas ocupa todavía un lugar importante el gasto de bolsillo asumido por los propios pobladores. La Red de Salud es asumida por el centro de salud de modalidad CLAS, que asume a la vez la responsabilidad de Cabecera de la Microrred Contamana (Ucayali), pero que no es una Unidad Ejecutora. Recibe apoyo de la empresa petrolera Maple Gas para la atención médica quincenal en la comunidad de Cannán; allí ser realiza una atención clásica de campaña médica sin realizar actividades en prevención y promoción de la salud. Se identificó un problema con EsSalud, algunos nativos que ya no trabajan en la empresa petrolera hace meses, no pueden ser inscritos en el SIS por persistir sus nombres en el sistema de gestión informatizado de EsSalud. 


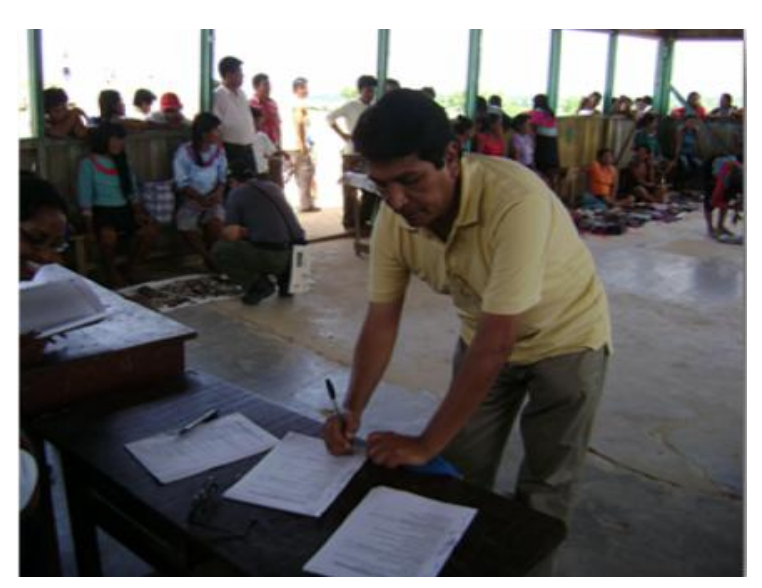

Figuras 3. Firma de acuerdos y Diálogo Intercultural en Salud con representantes Shipibos.

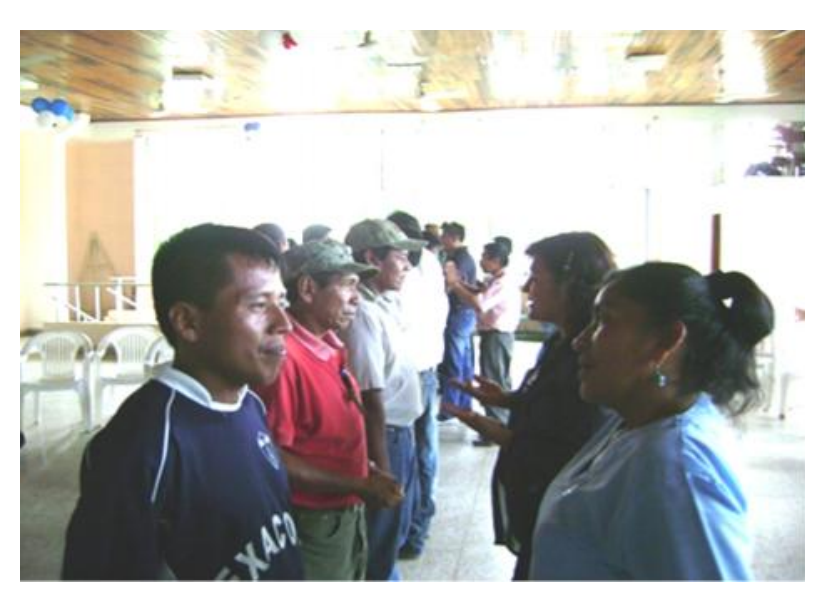

Figura 4. Desarrollo de diálogo intercultural. Cachiyacu y Nuevo Sucre Contamana - Loreto.

Gráfico № 1. Diseño general de la guía de operativización del DICS.

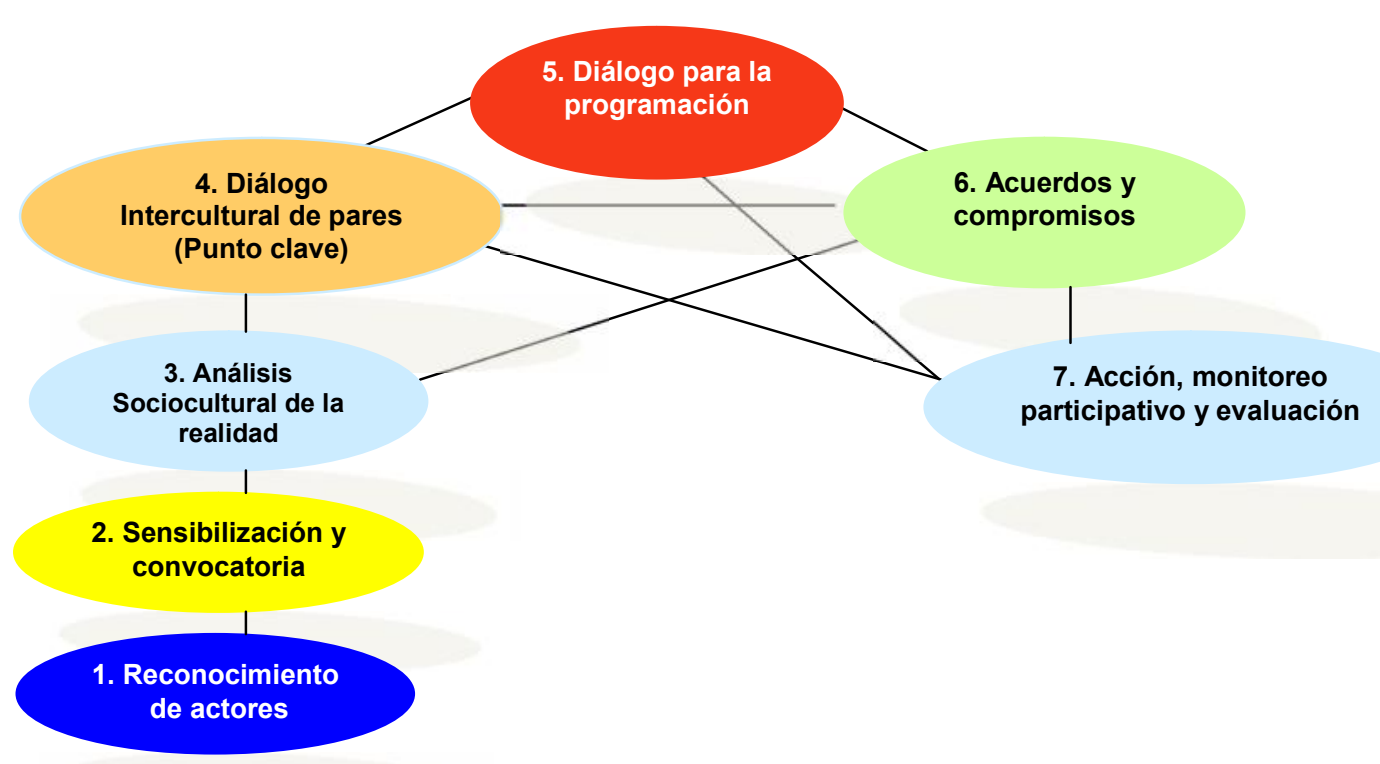

Basado en: P. Freire J. Piaget Ch. Maguerez ${ }^{y}$. Adecuado por Gualberto Segovia

El diálogo intercultural en salud nos permitió conocer la situación actual de algunos elementos esenciales de la atención primaria de la salud; con respecto a la accesibilidad y cobertura, que es el fundamento de un sistema de salud equitativo; encontramos que las poblaciones nativas de Canaán y Nuevo Sucre tienen brechas de acceso por falta de documento de identidad (DNI), que trae como consecuencia dificultades en la afiliación al Seguro Integral de Salud. Se ha observado falta de medios de comunicación para las referencias (bote a motor), deterioro de la radio para la comunicación, puente peatonal caído que dificulta el traslado cuando hay crecida del río, se conoce de problemas culturales por falta de adecuación de los servicios de salud, agregándose una insuficiente organización de la red de servicios de salud.

El primer contacto se refiere a que la atención primaria debe ser la puerta principal de entrada al sistema de salud; sin embargo, este elemento tiene marcadas deficiencias en contactar al usuario, quien siente "discriminación" y un trato no adecuado según su percepción sobre la atención en los servicios de salud. En tal sentido, se ha identificado que por falta de confianza en el Puesto de Salud de Canaán los pobladores se 
dirigen al CS Contamana, donde a veces no acceden a las consultas.

La atención debería ser integral, integrada y continua; esta deficiencia implica que la cartera de servicios no es suficiente para responder a las necesidades de salud de la población. Se encontró en la primera visita a dos pacientes con tuberculosis pulmonar (confirmada con los resultados de $\mathrm{BK}$ en esputo), que estuvieron siendo tratados para otra condición en sus domicilios, una paciente con úlcera crónica de mama izquierda con un tiempo de enfermedad de aproximadamente nueve meses. En la segunda visita se diagnosticó un tercer caso de tuberculosis pulmonar sin tratamiento alguno, se tuvo conocimiento del fallecimiento de una gestante joven madre de 30 años sin control prenatal y sin cobertura del Seguro Integral de Salud; luego, hace una semana, el fallecimiento de una niña de 10 años a causa de una mordedura de serpiente en la comunidad de Nuevo Sucre, identificándose problemas de falta de organización comunal para una referencia oportuna a Contamana, falta de movilidad (bote) para la referencia. Los servicios de salud no garantizan la continuidad de la atención por una comunicación deficiente entre los servicios de salud locales y el centro de salud de la provincia que es su centro de referencia inmediato.

Luego de los talleres se proponen soluciones consensuadas para mejorar el acceso de la población al $\mathrm{DNI}$ en coordinación con el gobierno local, RENIEC y los servicios de salud; mejorar el trato a la población indígena, reparar el puente en Nuevo Sucre y formar la red de promotores de salud con participación de las comunidades nativas y los servicios de salud entre otros. Los servidores de los servicios de salud reconocen y respetan el trabajo de la medicina tradicional y reconocen su uso prioritario en la población.

\section{DISCUSIÓN}

El diálogo intercultural en salud muestra su potencialidad para identificar el problema desde diferentes percepciones y permite plantear soluciones en el nivel local con los actores de la salud (personal de los servicios de salud, autoridades locales y agentes de la medicina tradicional), fortaleciendo la participación comunitaria e intersectorial, que representan los pilares de la atención primaria de la salud (APS). Un sistema de salud basado en la APS debe crear sinergias con otros sectores y actores para desempeñar una función muy importante, aunque no exclusiva, en la promoción de un desarrollo humano sostenible y equitativo. ${ }^{(7)}$

EI DICS promueve la interculturalidad y la articulación de la comunidad con el servicio de salud, la medicina tradicional con la medicina occidental o académica y la participación de la red comunitaria desde el inicio de la estrategia "crea un alto nivel de confianza, motivación y compromiso", en forma similar a otras experiencias. ${ }^{(8)}$

Finalmente, validamos en población amazónica el diseño de los pasos secuenciales de la guía de operativización del Diálogo Intercultural en Salud, y pueda ser implementada en el nivel nacional, como una estrategia del personal de los servicios de salud hacia una planificación local participativa y en el abordaje de los determinantes sociales de la salud en el nivel de la APS.

\section{Agradecimiento}

Al Centro Nacional de Salud Intercultural del Instituto Nacional de Salud por la oportunidad brindada.

\section{Correspondencia:}

Gualberto Segovia Meza

Correo electrónico: guaber2@yahoo.es

\section{REFERENCIAS BIBIOGRÁFICAS}

1. OMS. Comisión sobre Determinantes Sociales de la Salud. Subsanar las desigualdades en una generación. OMS 2009.

2. Defensoría del Pueblo. Conflictos Sociales y Gobernabilidad. Reporte mensual No 90. Agosto 2011.

3. CENSI - INS. Documento de trabajo de Guía Técnica del Diálogo Intercultural en Salud, 2011.

4. OIT. Convenio 169: Artículo 25.2 Parte V Seguridad Social y Salud. Sobre pueblos indígenas y Tribiales.

5. Comisión Multisectorial del Estado para el problema de derrame de Petróleo en Canaán de Cachiyacu y Nuevo Sucre, por la empresa Maple Gas. Informe de Octubre del 2011.

6. Acoontability Consul. Cronología de los hechos más importantes en Canaán de Cachiyacu y Nuevo Sucre. 8 California Street, Suite 650, San . Francisco, California, 94111, USA.

7. OPS/OMS. La Renovación de la Atención Primaria de Salud en las Américas. Documento de posición de la OPS/OMS julio 2007.

8. MINSA Nicaragua. USAID. UNICEF. Diálogo Intercultural para la Atención del Parto, entre la comunidad y el personal de salud. Marzo 2,009.

9. Díaz J. Algunos factores pedagógicos. Texto adaptado del artículo. La Transferencia de Tecnología Apropiada al Pequeño Agricultor (Bordenave, J.E.D., Revista Interamericana de Educación de adultos 3(12)

Recibido: 15/02/2012

Aceptado para publicación:15/06/2012 